\title{
Function and molecular mechanism of a nucleolar protein LYAR in embryonic stem cells
}

\author{
Beibei Wang ${ }^{1}$, Hui $\mathrm{Li}^{1}$, Rui $\mathrm{Lu}^{1}$, Ying $\mathrm{Jin}^{1}$ \\ ${ }^{1}$ The Key Laboratory of Stem Cell Biology, Institute of Health Sciences, Chinese Academy of Sciences, Shanghai 200025, China
}

Embryonic stem (ES) cells are attractive cell sources for regenerative medicine. However, they also have tumorlike properties. Therefore, it is crucial to understand the mechanisms that control their growth and propensity to form tumors. Here, we show that down-regulation in expression of a nucleolar protein LYAR in mouse ES cells significantly reduces cell proliferation, anchorage-independent growth, and teratoma formation. LYAR is highly expressed in undifferentiated ES cells, but its expression level is reduced with differentiation. Our data show that endogenous LYAR forms protein complex with Nucleolin and colocalizes with it in nucleolus of ES cells. Strikingly, the steady-state protein level of Nucleolin is decreased when LYAR expression is knocked down, whereas its mRNA level remains unchanged. Similarly, the down-regulation of Nucleolin expression in ES cells substantially reduces cell proliferation. In addition, we confirm that LYAR is able to stabilize Nucleolin by preventing its autodegradation. We conclude that LYAR is required for cell proliferation and the tumor-like growth properties in ES cells and its interaction with Nucleolin is the major mechanism for its function. The work describes a novel nucleolar mechanism to control ES cell proliferation in vitro and teratoma formation in vivo.

Keywords: embryonic stem cells, proliferation, protein stability

Cell Research (2008) 18:s7. doi: 10.1038/cr.2008.97; published online 4 August 2008

Correspondence: Ying Jin

E-mail: yjin@sibs.ac.cn

Ying Jin, MD, PhD, is currently a Principal Investigator at the Institute of Health Sciences, Shanghai institutes for Biological Sciences, Chinese Academy of Sciences (CAS) and Shanghai Jiaotong University School of Medicine. She is also Director of the Key Laboratory of Stem Cell Biology of CAS and Director of Shanghai Stem Cell Institute, Shanghai JiaoTong University. Over the past several years, Dr Jin has been directed at an understanding the molecular and cellular basis of mammalian embryonic development. Using embryonic stem cells as a model system, her lab has been exploring protein-protein, protein-DNA interaction network, dissecting important protein complexes and pathways involved in the proliferation and differentiation of ES cells, in particular, Oct-4 functions. In addition, their research also includes derivation of human and mouse ES cell lines and directed differentiation of ES cell into neural lineage. Dr Jin has been ISSCR member since year 2003 and served as ISSCR Program Committee member in 2006. 